\title{
Feasibility of screening for diabetic retinopathy at an Australian pathology collection service: a pilot study
}

\section{Melanie F Larizza \\ BBSc, GradDip(Sec) Research Assistant \\ Lauren A Hodgson BSc(Hons), Research Assistant \\ Eva K Fenwick MA, BA(Hons) Research Fellow \\ Ryo Kawasaki PhD, MD, MPH, Research Fellow \\ Ralph Audehm MB BS, \\ Clinical Director \\ Jie Jin Wang \\ PhD, MB BS, \\ MMed(OphthalPath) Senior Research Fellow \\ Tien $\mathbf{Y}$ Wong PhD, FRCEEd, MMed Senior Research Fellow ${ }^{1,3}$ \\ Ecosse L Lamoureux \\ PhD, BEd, MApplSci Senior Research Fellow \\ 1 Centre for Eye Research Australia, Melbourne, VIC. \\ 2 Dianella Community Health, Melbourne, VIC \\ 3 Singapore Eye Research \\ Institute, Singapore. \\ ecosse@unimelb.edu.au}

MJA 2013; 198: 97-99 doi:10.5694/mjal2.11121

Editorial p 69 Research p 93 arly detection of diabetic retinopathy (DR) and timely treatment are integral to preventing vision-threatening DR in people with diabetes. ${ }^{1,2}$ The National Health and Medical Research Council (NHMRC) guidelines for the management of DR recommend biannual screening in those with no previous diagnosis and annual screening (at least) for those who have had a diagnosis of $\mathrm{DR}^{3}$ However, population-based studies show that $30 \%-50 \%$ of Australians with diabetes do not undergo adequate screening. ${ }^{4-6}$ Despite the implementation of successful national screening programs worldwide, ${ }^{7,8}$ comprehensive DR screening strategies are lacking in Australia. We propose a novel screening strategy using pathology collection centres (PCCs) as screening sites. A PCC offers the potential to opportunistically screen people with diabetes for $\mathrm{DR}$, as up to $90 \%$ of people with diabetes regularly attend PCCs for diabetes-related tests. $^{9}$

The purpose of this pilot study was to test the feasibility of our new screening model in one PCC. The specific aims of this study were to determine (1) the proportion of patients who do not participate in biannual screening; (2) the proportion of patients who accept DR screening while attending a PCC; (3) the proportion of gradable images; (4) patient acceptance of the screening model; and (5) the effectiveness of the followup pathway with treating doctors.

\section{Methods}

\section{Participants}

Patients who attended an urban PCC (St Vincent's Pathology, Craigieburn, Melbourne, Victoria) from 1 September 2009 to 15 March 2010 were invited to receive a free DR screening test if they had type 1 or type 2 diabetes (self-reported), spoke English, were at least 12 years of age and

\begin{abstract}
Abstrac
Objective: To evaluate the feasibility of a novel diabetic retinopathy (DR) screening model using a pathology collection centre (PCC) as a screening site.

Design and setting: Cross-sectional pilot study conducted in one urban PCC in Victoria between 1 September 2009 and 15 March 2010. Trained pathology staff screened participants for DR using undilated, non-stereoscopic colour fundus photography.
\end{abstract}

Participants: Participants were patients who spoke English, were aged at least 12 years, had type 1 or type 2 diabetes, and did not undertake biannual DR screening.

Main outcome measures: Proportion of patients who do not participate in biannual DR screening; proportion of patients who accept DR screening while attending a PCC; proportion of gradable images; patient acceptance of the screening model; and effectiveness of the follow-up pathway.

Results: Over 6 months, 289 English-speaking patients with diabetes attended the PCC, of whom 99 (34.3\%) had not undertaken biannual DR screening. Of these, 93 (93.9\%) accepted our screening service. Overall, retinal images were sufficient for interpretation in 88 patients (94.6\%) and the median time for photography was 6 minutes (interquartile range, 10 minutes). Two, eight and six cases of minimal, mild and moderate non-proliferative DR (NPDR) in the worst eye were found, respectively. Dissemination of screening results to treating doctors and patients was found to be suboptimal and will need to be revised.

Conclusion: DR screening in one urban PCC appears to be a feasible alternative for diabetic patients who do not undertake biannual screening for DR.

did not undertake biannual DR screening.

\section{Protocol}

Patients' sociodemographic details were collected, including diabetes type, duration of diabetes and reasons for not having biannual DR screening. Presenting distance binocular visual acuity was assessed with an eye chart. In a darkened room, two $45^{\circ}$ nonmydriatic photographs were taken for each eye, centred on the optic disc and macula. ${ }^{10}$ Images were sent to the Centre for Eye Research Australia for assessment. Patients were asked to grade their satisfaction with the photographic procedure, on a 5-point Likert scale, from "very satisfied" to "very dissatisfied", and the degree to which our screening service would give them more control over the management of their diabetic eye care, from "not at all" to "a considerable degree". Questionnaire items were based on published literature. ${ }^{11-13}$ Images were graded by trained graders and validated by an ophthalmologist. The presence of DR was defined with the Early Treatment Diabetic Retinopathy Study severity scale ${ }^{10}$ and classified as none, minimal nonproliferative DR (NPDR), mild NPDR, moderate NPDR, severe NPDR, proliferative $\mathrm{DR}$ (PDR) and high-risk PDR. ${ }^{14}$ Follow-up recommendations were made in accordance with the NHMRC guidelines. ${ }^{3}$

A report outlining the results of the screening and follow-up recommendations was sent electronically to the patient's treating doctor by the pathology organisation within 2 weeks of screening. Two weeks after the distribution of reports, we contacted $50 \%$ of patients at random for a follow-up telephone interview to verify whether the treating doctor had received and shared the outcome of the screening test with the patient and provided any recommended referrals. Box 1 shows a flow chart of the testing protocol.

Ethics approval for this study was provided by the Royal Victorian Eye and Ear Hospital Human Research and Ethics Committee $(08 / 838 \mathrm{H})$ and participants gave written informed consent. 


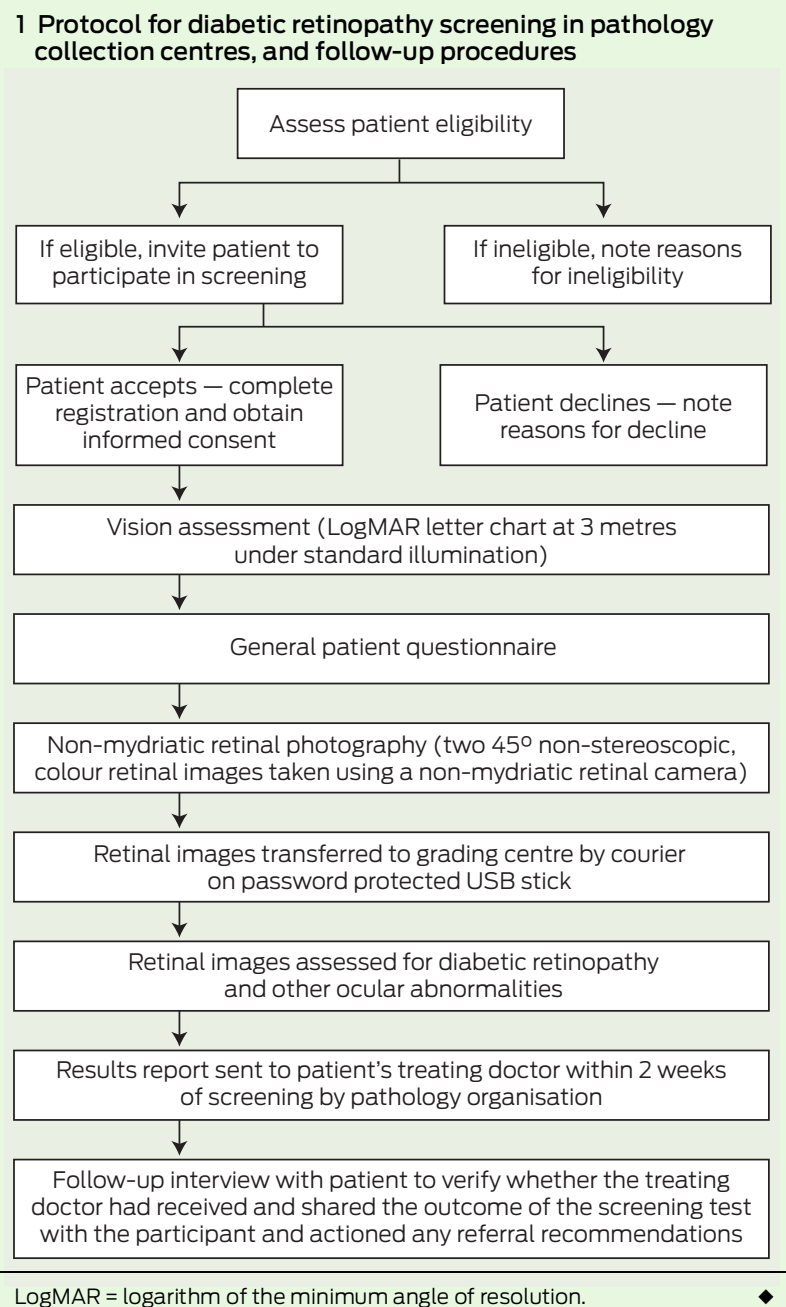

LogMAR = logarithm of the minimum angle of resolution results via their GP were presented as $n$ and $\%$. All statistics were obtained with Stata version 11 (StataCorp).

\section{Results}

\section{Feasibility of DR screening in a PCC}

Over 6 months, 1474 patients visited the PCC. Four hundred and eleven patients $(27.9 \%)$ had self-reported diabetes, and of those, 289 (70.3\%) spoke English. Of the patients who spoke English, 99 had not had biannual DR screening (34.3\%), and of those, 93 (93.9\%) accepted our invitation to be screened for DR. Six patients declined, giving the reason that they were too busy (two patients); considered DR screening unnecessary (two patients) or had insufficient motivation (one patient). One patient did not provide a reason.

The mean age of the 93 patients who accepted our invitation for screening was $54.8 \pm 12.2$ years (range, $24-84$ years), 50 were male $(53.8 \%)$, and 81 had type 2 diabetes $(87.1 \%)$ and a median known diabetes duration of 4.0 years (IQR, 6.0 years). Reasons given for not having biannual DR screening included being too busy (25 patients; 26.9\%), being recently diagnosed with diabetes (10 patients; $10.8 \%$ ), being unaware of the need for DR screening (13 patients; 14.0\%), insufficient motivation (four patients; $4.3 \%$ ), lack of advice (three patients; $3.2 \%$ ), "don't know" (three patients; $3.2 \%$ ), "forgot" (two patients; $2.2 \%$ ), health reasons (two patients; $2.2 \%$ ) and cost (one patient; 1.1\%); six patients $(6.5 \%)$ did not provide a reason. Twenty-four patients (25.8\%) reported that they were due for screening at the time of the study.

We were able to obtain two gradable images for 138 (74.2\%) of the 186 eyes examined (ie, well centred images with good focus and illumination). The interpretation of images of 41 eyes $(22.0 \%)$ was limited because of poor image quality of one or both fields as a result of small pupils, media opacity, poor fixation or the absence of one field. The images of seven eyes $(3.8 \%)$ were not able to be graded. Inter- and intra-grader reliability for any referrable level of DR were assessed by a $\kappa$ statistic and found to be 1 . Screening results and referral recommendations for the 93 patients are presented in Box 2. Sixteen patients $(17.2 \%)$ were diagnosed with DR in at least one eye. The median time for photography was 6 minutes (IQR, 10 minutes). Most patients were "satisfied" to "very satisfied" with the photographic procedure (91 patients; $97.8 \%)$ and only two patients (2.2\%) reported that the screening service would not give them more control over the management of their diabetic eye care.

\section{Participant follow-up}

Thirty-seven of 47 patients (78.7\%) who were randomly selected for a follow-up telephone interview had visited their GP by the time of the interview. Of those, five (13.5\%) discussed the screening results with their GP. Four of these five patients had been advised during this study to have a follow-up assessment with an eyecare professional. However, only two patients out of the five received a referral to an eye care professional from their GP.

\section{Discussion}

DR screening in one PCC was well accepted by diabetic patients who do not participate in biannual DR screening, and was successful in terms of image acquisition. One-third of English-speaking patients with diabetes had not undertaken biannual DR screening, and almost all of the patients (93.9\%) accepted our DR screening service. The process of sending results to treating doctors and patients was suboptimal and needs revision.

Our finding that a third of diabetic patients had not undertaken biannual DR screening is similar to non-adherence rates found in other studies. ${ }^{4,6,15}$ Being busy, being recently diagnosed with diabetes and being unaware of the need for DR screening were identified as barriers to DR screening that were largely overcome by our screening model in this sample. Our finding that $17.2 \%$ of patients had DR is also comparable with the findings of other DR screening studies. ${ }^{15,16}$ Interestingly, we found no cases of PDR; however, the median known duration of diabetes was 4 years, which might account for this. Although our pro- 
2 Results of diabetic retinopathy screening and referral recommendations for 93 patients with type 1 or type 2 diabetes

\begin{tabular}{llc} 
Screening result & Referral recommendation & Participants \\
\hline Visual acuity $\geqslant 6 / 9.5$ in best eye and no significant abnormality & Routine screening in 2 years & 40 \\
Visual acuity (<6/9.5) in best eye and no significant abnormality & Follow-up assessment in 3-6 months & 22 \\
Minimal NPDR in worst eye & Follow-up assessment in 12 months & 2 \\
Mild NPDR in worst eye & Follow-up assessment within 3-6 months & 7 \\
Moderate NPDR & Follow-up assessment within 4 weeks & 5 \\
Moderate NPDR and other significant conditions* & Follow-up assessment within 4 weeks & 1 \\
Other significant abnormality & Follow-up assessment within 1-6 months & 11 \\
Images ungradable in one or both eyes & Follow-up assessment within 4 weeks & $5^{\dagger}$
\end{tabular}

NPDR = non-proliferative diabetic retinopathy. * Other significant conditions included signs of age-related macular degeneration and glaucoma (eg, cupdisc ratio 0.6 ), vascular abnormalities and indications of branch retinal vein occlusion. $†$ One participant had ungradable images in one eye and mild NPDR in the other eye.

gram successfully enrolled patients with diabetes who did not participate in biannual DR screening, our model needs to be revised to ensure that the GP and patient are well informed of the screening results to help patients make informed decisions about their diabetic eye care.

Strengths of this study include a prospective study design and standardised DR evaluation from retinal photographs. However, the generalisability of our findings may be limited. Our findings are based on English-speaking patients who attended one urban PCC over a 6month period. Nonetheless, the findings of this study inform a current longitudinal multicentre study, involving English-speaking and nonEnglish-speaking people with diabetes at 10 urban and rural PCCs across Victoria, to determine if acceptance of our DR screening model remains high across multiple centres and is sustained over a longer period of time. We also plan to determine the number of people with positive results of screening for DR who follow up on their referral. Finally, the economic effectiveness of this model will be investigated in the multicentre study. Although not explored in this pilot study, major costs of this study included the camera and laptop (approximately \$30000), the grading of retinal images (about $\$ 4$ per patient) and the employment of a third pathology staff member to cover the workload of staff administering DR screening (about \$20 per hour). Funding for this model could be supported by governments and other decisionmakers (eg, private health insurance companies).

In summary, DR screening in one urban PCC by means of non-mydriatic retinal photography was considered a feasible way to screen for DR in patients with diabetes who do not adhere to biannual DR screening.

Acknowledgements: We would like to acknowledge our funding sources - the NHMRC Centres for Clinical Research Excellence (NHMRC grants 454461, 529902 and 529923), and Diabetes Australia - Vic. Other research collaborators included St Vincent's Pathology and the Royal Victorian Eye and Ear Hospital. The Centre for Eye Research Australia receives Operational Infrastructure Support from the Victorian government.

Competing interests: No relevant disclosures.

Received 18 Jul 2012, accepted 21 Nov 2012.

1 Ferris FL. How effective are treatments for diabetic retinopathy? JAMA 1993; 269: 1290-1291.

2 Rohan TE, Frost CD, Wald NJ. Prevention of blindness by screening for diabetic retinopathy: a quantitative assessment. BMJ 1989; 299: 11981201

3 National Health and Medical Research Council. Guidelines for the management of diabetic retinopathy. Canberra: Australian Commonwealth Government, 2008. http:// www.nhmrc.gov.au/guidelines/publications/dil5 (accessed Jul 2012).

4 McCarty CA, Lloyd-Smith CW, Lee SE, et al. Use of eye care services by people with diabetes: The Melbourne Visual Impairment Project. Br J Ophthalmol 1998; 82: 410-414.

5 Muller A, Vu HT, Ferraro JG, et al. Utilization of eye care services in Victoria.Clin Experiment Ophthalmol 2006; 34: 445-448.
6 Tapp RJ, Zimmet PZ, Harper CA, et al. Diabetes care in an Australian population: frequency of screening examinations for eye and foot complications of diabetes. Diabetes Care 2004; 27: 688-693.

7 Arun CS, Al-Bermani A, Stannard K, Taylor R. Long-term impact of retinal screening on significant diabetes-related visual impairment in the working age population. Diabet Med 2009; 26: 489-492.

8 Bäcklund LB, Algvere PV, Rosenqvist U. New blindness in diabetes reduced by more than onethird in Stockholm County. Diabet Med 1997; 14: 732-740.

9 National Institute of Clinical Studies. Evidencepractice gaps report volume l: a review of developments 2004-2007. Canberra: National Health and Medical Research Council, 2008.

10 Grading diabetic retinopathy from stereoscopic color fundus photographs - an extension of the modified Airlie House classification. ETDRS report number 10. Early Treatment Diabetic Retinopathy Study Research Group. Ophthalmology 1991; 98 (5 Suppl): 786-806.

11 Eiser JR, Eiser C, Riazi A, et al. Screening for diabetic retinopathy is well received by patients and may improve self-management intentions. Diabetic Med 2001; 18: 835-841.

12 Okoli U, Mackay K. An evaluation of diabetic retinopathy screening models. J Public Health Med 2002; 24: 190-195.

13 Spurling GKP, Askew DA, Hayman NE, et al. Retinal photography for diabetic retinopathy screening in Indigenous primary health care: the Inala experience. Aust NZJ Public Health 2010;34 Suppl 1: S30-S33.

14 Diabetic retinopathy study. Report number 6. Design, methods, and baseline results. Report number 7. A modification of the Airlie House classification of diabetic retinopathy. Prepared by the Diabetic Retinopathy. Invest Ophthalmol Vis Sci 1981; 21: 1-226.

15 Harper CA, Livingston PM, Wood C, et al. Screening for diabetic retinopathy using a nonmydriatic retinal camera in rural Victoria. Aust NZ J Ophthalmol 1998; 26: 117-121.

16 Levy J, Lifshitz T, Goldfarb D, et al. Screening for diabetic retinopathy with a mobile non-mydriatic digital fundus camera in Southern Israel. Isr Med Assoc J 2011; 13: 137-140. 